\title{
Feature Extraction of Elderly Signals based on Bicoherence Estimation for Automated Medical Diagnosis System
}

\author{
Ji Yeoun Lee \\ Department of Biomedical Engineering, Jungwon University \\ Donbu-ri \#5, Goesan-eup, Goesan-gun, Chungcheongbuk-do 367-805, South \\ Korea \\ jylee@jwu.ac.kr
}

\begin{abstract}
Physiological changes in the vocal fold due to aging may change the pitch of the voice and the elderly signal can be automatically distinguished from the normal signal through various analyses. In case of most smart biomedical devices, the elderly voices have been neglected due to optimization which does not take into account the elderly. The objective of this study is to find the parameters that can distinguish the elderly signal. This paper suggests the mean of normalized skewness, the mean of normalized kurtosis, the ratio of the normalized skewness and kurtosis (RSK), and maximum bicoherence (MB) based on the linear predictive coding (LPC) residual. The parameters are based on the higherorder statistics (HOS) of the time and frequency domain. The mean of normalized skewness and MB parameter were useful and meaningful in an analysis between normal and elderly signals because they showed p-values <.05. In particular, it was believed that $M B$ was very significant for the classification between two signals. And the mean of normalized skewness, RSK, and $M B$ were significant for the objective classification among normal, female elderly, and male elderly signals. In most cases, MB is specially performs better statistically than do the other parameters. To improve the performance of speech interface in automated medical diagnosis system, an analysis of elderly signal is of great interest. These results will contribute to provide an easy access means for the elderly. Future investigations will incorporate multiple classification methods to implement more reliable detector for automated medical diagnosis system.
\end{abstract}

Keywords: Elderly signal, Disorder voice, Bicoherence, Higher-order statistics, Linear predictive coding, Medical diagnosis system

\section{Introduction}

The computerized smart training devices with speech interface have been made actively for education and community welfare facilities [1]. However, in case of most smart medical devices, the elderly voices have been neglected from the technology due to interface which does not take into account the elderly [2]. Speech interface that supports a smart device is currently using an optimized method based on the average speech pattern of young adults, middle-aged, and elderly people. If it is a little larger deviation from the standard, it may result in a phenomenon that degrades the performance of voice analysis and recognition [2]-[4].

The aging of the body beginning to move into adulthood brings morphological changes in the tissue of the vocal cords and larynx structure that is directly relevant to voice. Therefore elderly voice is to be understood in conjunction with the acoustic properties of the sound by the larynx change according to aging. It means voice changes caused by natural aging process as a result of the drying of the mucosal, the reduced flexibility of vocal ligament, and the nervous fibers atrophy, necrosis [5]-[6]. That is, anatomy and 
physiological changes in the larynx and the vocal cords may change the pitch of the voice and it is measured by fundamental frequency (F0). In conclusion, elderly voice is distinguished from the normal voice and it belongs to a part of speech disorder.

Although many studies related to elderly signals have been published, they are based on the acoustic analysis of the voice samples including jitter and shimmer [3][7]-[9]. However, since these parameters are based on the fundamental frequency, a very reliable pitch detection algorithm is necessary to measure voicing irregularities in elderly signals [10][11]. The perturbation analysis has been found to be sensitive to pitch variations in analysis tools such as multi-dimensional voice profile (MDVP), CSpeech, and TF32. Recently, Lee proposed the moving window method as an objective and reliable method of sample selection for Korean elderly signals. It utilized minimum perturbation value of perturbation measures such as jitter (\%), shimmer (\%), and SNR (dB) to extend perturbation analysis. It also compared minimum perturbation with average values of the acoustic parameters and investigated the impact of the moving window on perturbation measures generated from elderly signal samples [12].

This paper utilizes higher-order statistics (HOS) that is nothing to do with pitch estimation. Many studies have applied HOS to disordered voices since Alonso et al.'s publication on automatic detection of voice pathologies by HOS-based parameters [13][18]. Further, the combination of HOS analysis and the linear predictive coding (LPC) residual may help to effectively construct important information to distinguish the signal types of disordered voices [17]-[18]. Although HOS analysis holds promise as one possible method of distinguishing between normal/pathological voices and signal type classification [14]-[16], no studies have applied HOS analysis to analyze elderly voices. Therefore, novel HOS- based parameters estimated in frequency domain for analysis of elderly voices are proposed in this paper.

In this study, an author analyzes the speech of the elderly and finds the necessary correction factor by comparing the normal voices of young adults and middle-aged people. The HOS-based parameters in the time and frequency domains are presented in this paper in order to analyze the elderly voices and to improve the classification performance. This will enhance the speech recognition performance of existing smart medical systems for the elderly. This is also expected to help provide an easy access means of the elderly and people with disabilities who were excluded from the rapid socialization.

\section{Research Method}

\subsection{Database}

The elderly signal samples were collected in The Speech Information Technology\&Industry Promotion Center (SiTEC). The database includes the elderly signals of 20 Korean subjects ( 10 female and 10 male) ranging in age from 70 to 80 years. Two sentences were used in this study. The normal signal samples were collected in the speech clinic, Otorhinolaryngology of Gangnam Severance Hos pital. The database includes the normal signals of 10 Korean subjects (10 female) ranging in age from 20 to 30 years. Silence sections are manually deleted using information such as structure of waveform and spectrum. The signal samples were sampled at $22.5 \mathrm{kHz}$ and the information is detailed in Table 1. 
Table 1. Database Information

\begin{tabular}{|c|c|c|c|c|}
\hline & Sex & Number & Age & Korean sentences \\
\hline \multirow[t]{2}{*}{$\begin{array}{l}\text { Elderly } \\
\text { signals }\end{array}$} & Female & 10 & $70-79$ & $\begin{array}{l}\text { 1. 그때 누가 그녀의 } \\
\text { 책상 } \\
\text { 다가왔다. }\end{array}$ \\
\hline & Male & 10 & $70-78$ & $\begin{array}{l}2 . \quad \text { 그때 웬 낯선 } \\
\text { 사람이 다가와 } \\
\text { 물었다. }\end{array}$ \\
\hline \multirow[t]{2}{*}{$\begin{array}{c}\text { Normal } \\
\text { signals }\end{array}$} & Female & 10 & $20-30$ & $\begin{array}{l}\text { 1. 무엇보다도 산에 } \\
\text { 오를 땐 더욱 더 그 } \\
\text { 빼어난 아름다움이 } \\
\text { 느껴진다. }\end{array}$ \\
\hline & Male & $\mathrm{X}$ & $\mathrm{X}$ & $\mathrm{X}$ \\
\hline
\end{tabular}

\subsection{LPC Analysis}

In previous study, Lee et al. introduced the LPC characteristics as acoustic parameters to classify normal and pathological signals [17]-[20]. They suggested HOS-based parameters obtained from the LPC residual for voice-quality assessment. The presented techniques demonstrated that it is possible to evaluate the extent of larynx diseases. Therefore, LPC analysis was introduced in this study to investigate its ability to automatically analyze the elderly signal.

Figure 1 shows the LPC residuals estimated for normal and elderly signals. Figure 1 (a) shows the LPC residuals estimated in normal signals. Then it is zoomed out as Figure 1 (b) to compare LPC residuals of elderly signals with same amplitude unit. Amplitudes of LPC residuals estimated in elderly signals are larger than those of normal signal. Only some of the residuals are harmonically related in Figure 1 (c) and (d). It can be seen that the residual errors tend to be high because of pulse regularity and noise between the signals in elderly voices. Therefore, the use of LPC residuals may yield information regarding the abnormal movement of vocal folds and turbulence noise, which is useful for elderly signals classification. 




Figure 1. LPC Residuals Estimated for Normal and Elderly Signals

\subsection{HOS Analysis}

HOS analysis in the time domain has shown promise as a classification index for pathological signals and also has the advantage of not requiring a periodic or quasiperiodic signal to enable reliable analysis [7-9].

The normalized skewness $\left(\gamma_{3 t}\right)$ and kurtosis $\left(\gamma_{4 t}\right)$ are extracted in-frame, as shown in Equation (1):

$$
\gamma_{3 t}=\frac{\sum_{n=1}^{N} x_{t}^{3}(n)}{\left[\sum_{n=1}^{N} x_{t}^{2}(n)\right]^{1.5}}, \quad \gamma_{4 t}=\frac{\sum_{n=1}^{N} x_{t}^{4}(n)}{\left[\sum_{n=1}^{N} x_{t}^{2}(n)\right]^{2}}
$$

where $\mathrm{x}(\mathrm{n})$ is the speech signal sample value of the $t^{\text {th }}$ frame, and $N$ is the number of samples.

Then, the means of the normalized skewness and kurtosis are applied for this study.

$$
\overline{\gamma_{3}}=\frac{1}{N} \sum_{t=1}^{N} \gamma_{3 t}, \quad \overline{\gamma_{4}}=\frac{1}{N} \sum_{t=1}^{N} \gamma_{4 t}
$$

The proposed parameter is the ratio of the normalized skewness and kurtosis (RSK) as indicated in Equation (3): 


$$
R S K=\frac{\left(\overline{\gamma_{3}}\right)^{4}}{\overline{\left(\gamma_{4}\right)^{3}}}
$$

HOS analysis in the frequency domain is mainly applied by using a bispectrum or trispectrum. A bispectrum is a function of two frequencies, whereas a trispectrum is a function of three frequencies. Both spectrums contain complex values. This investigation utilized the bispectrum shown in Equation (4):

$$
B\left(f_{1}, f_{2}\right)=\sum_{\tau_{1}=-\infty}^{\infty} \sum_{\tau_{2}=-\infty}^{\infty} C_{3 x}\left(\tau_{1}, \tau_{2}\right) e^{-j \sum_{i=1}^{2} f i \tau_{i}}
$$

where $C_{3 x}\left(\tau_{1}, \tau_{2}\right)$ are the third-order cumulants and are defined by Equation (4):

$$
C_{3 x}\left(\tau_{1}, \tau_{2}\right)=E\left\{x(t) x\left(t+\tau_{1}\right) x\left(t+\tau_{2}\right)\right\}
$$

Given estimates of the power spectra and bispectrum, the bicoherence can be estimated as indicated in Equation (5):

$$
\text { bic }_{\text {auto }}\left(f_{1}, f_{2}\right)=\frac{S_{x x x}\left(f_{1}, f_{2}\right)}{\sqrt{S_{x x}\left(f_{1}\right) S_{x x}\left(f_{2}\right) S_{x x}\left(f_{1}+f_{2}\right)}}
$$

where $S_{x x}$ and $S_{x x x}$ are the power spectrum and bispectrum, respectively.

The proposed maximum bicoherence (MB) parameter results from the bicoherences. Thus the MB parameter is defined by Equation (7):

$$
\max (\text { Bicoherence })=\max \left(\sum_{f_{1}=0}^{\operatorname{dim}_{x}-1} \sum_{f_{2}=0}^{\operatorname{dim}_{y}-1} \operatorname{bic}\left(f_{1}, f_{2}\right)\right)
$$

where the dimensions of the bicoherence value are $\operatorname{dim}_{x}$ and $\operatorname{dim}_{y}$.

\section{Results and Analysis}

\subsection{Distributions of HOS-Parameters Estimated In Time Frequency}

Figure 2 shows the distributions of the normalized skewness and kurtosis estimated from the LPC residuals in normal and elderly signals. The signals were divided into frames of $20 \mathrm{~ms}$ with an overlap of $10 \mathrm{~ms}$ to extract an LPC residual. Each frame was then windowed by using a hamming window. The reflection coefficients $\left(12^{\text {th }}\right.$ order) were calculated from the $12^{\text {th }}$-order autocorrelation coefficients by using the Levinson-Durbin algorithm. The output of the all-zero analysis filter was the residual signal.

Figure 2(a) and (b) present the normalized skewness and kurtosis in the form of box plots to provide better visualizations of normal and elderly signals. As may be seen from Table 2, the normalized skewness estimated in normal signals tends to be greater than zero. That is, the mean of normal signals (Female) is 0.233 and the means of elderly signals (Female) and (Male) are -0.005 and -0.173 , respectively. Also the mode of normal signals (Female) is 0.34 . There is a difference between the distribution of the normalized skewness of normal and elderly signals. In case of the normalized kurtosis, one estimated in elderly signals tend to be larger than one estimated in normal signals. That is, the mean of normal signals (Female) is 6.583 and the means of elderly signals (Female) and (Male) are 7.229 and 7.103, respectively. But the mode of normal signals (Female) is 1.93 and the means of elderly signals (Female) and (Male) are 1.97 and 1.85, respectively. Also the median of normal signals (Female) is 4.074 and the means of elderly signals (Female) and (Male) are 4.260 and 4.040, respectively. So there is little difference between the distribution of the normalized kurtosis of normal and elderly signals. 
In particular, in normal signals, the distributions of the normalized skewness and kurtosis tend towards higher values and are slightly broader than those of elderly signals. Overall, it is evident that the normalized skewness is sufficiently distinct to analyze between the normal and elderly signals and can be used as a basis for automatic classification of the normal and elderly signals.

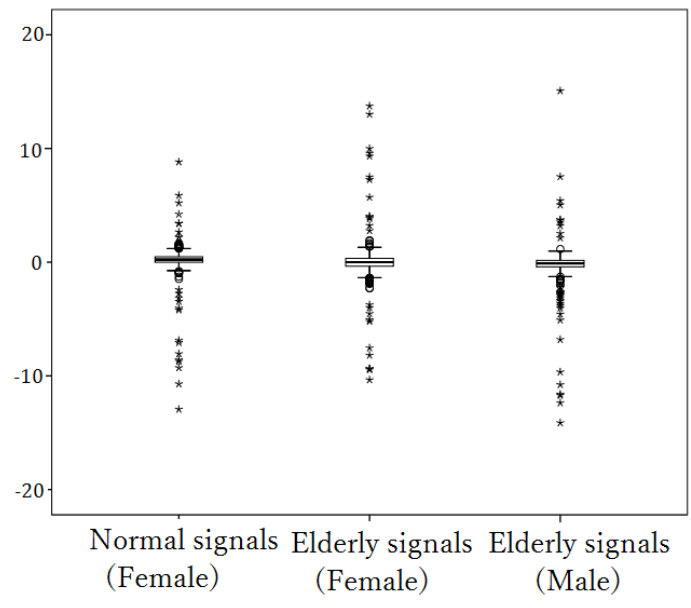

(a) The distribution of the normalized skewness

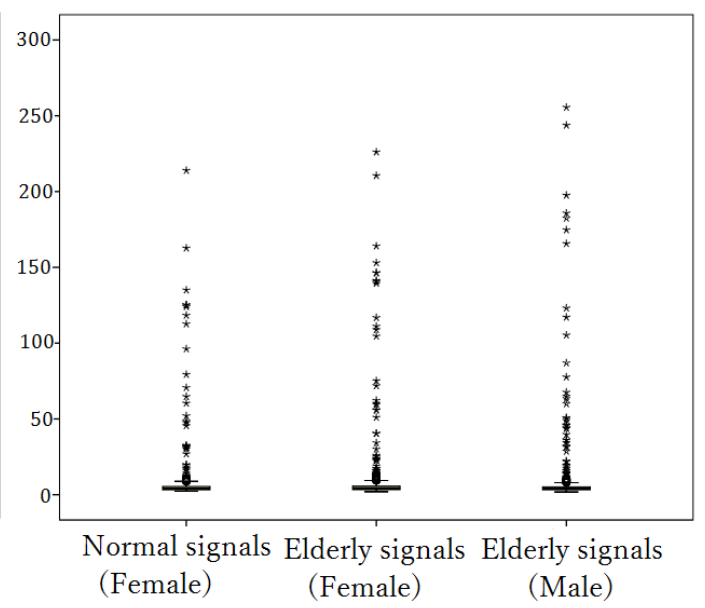

(b) The distribution of the normalized kurtosis

Figure 2. Distributions of the Normalized Skewness and Kurtosis

Table 2. Statistics of the Normalized Skewness and Kurtosis

\begin{tabular}{|c|c|c|c|c|}
\hline \multicolumn{2}{|c|}{ The normalized skewness } & $\begin{array}{c}\text { Normal signals } \\
\text { (Female) }\end{array}$ & $\begin{array}{c}\text { Elderly signals } \\
\text { (Female) }\end{array}$ & $\begin{array}{c}\text { Elderly signals } \\
\text { (Male) }\end{array}$ \\
\hline \multicolumn{2}{|c|}{ Mean } & 0.233 & -0.005 & -0.173 \\
\hline \multicolumn{2}{|c|}{ Median } & 0.221 & 0.006 & -0.094 \\
\hline \multicolumn{2}{|c|}{ Mode } & 0.34 & -10.35 & -14.13 \\
\hline \multicolumn{2}{|c|}{ Range } & 29.84 & 24.09 & 29.21 \\
\hline \multicolumn{2}{|c|}{ Minimum value } & -14.65 & -10.35 & -14.13 \\
\hline \multicolumn{2}{|c|}{ Maximum value } & 15.20 & 13.74 & 15.07 \\
\hline \multirow[t]{3}{*}{ Percentile } & 25 & -0.024 & -0.353 & -0.412 \\
\hline & 50 & 0.221 & 0.006 & -0.094 \\
\hline & 75 & 0.516 & 0.335 & 0.176 \\
\hline \multicolumn{2}{|c|}{ The normalized kurtosis } & $\begin{array}{c}\text { Normal signals } \\
\text { (Female) }\end{array}$ & $\begin{array}{l}\text { Elderly signals } \\
\text { (Female) }\end{array}$ & $\begin{array}{c}\text { Elderly signals } \\
\text { (Male) }\end{array}$ \\
\hline \multicolumn{2}{|c|}{ Mean } & 6.583 & 7.229 & 7.103 \\
\hline \multicolumn{2}{|c|}{ Median } & 4.074 & 4.260 & 4.040 \\
\hline \multicolumn{2}{|c|}{ Mode } & 1.93 & 1.97 & 1.85 \\
\hline \multicolumn{2}{|c|}{ Range } & 256.70 & 224.10 & 253.71 \\
\hline \multicolumn{2}{|c|}{ Minimum value } & 1.93 & 1.97 & 1.85 \\
\hline \multicolumn{2}{|c|}{ Maximum value } & 258.63 & 226.06 & 255.56 \\
\hline \multirow[t]{3}{*}{ Percentile } & 25 & 3.206 & 3.289 & 3.290 \\
\hline & 50 & 4.074 & 4.260 & 4.040 \\
\hline & 75 & 5.568 & 5.742 & 5.182 \\
\hline
\end{tabular}

Figure 3(a) and (b) describe the distributions of the means of the normalized skewness and kurtosis in the form of box. In the distribution of Figure 3 (a), the means of the normalized skewness estimated from normal signals tend to be greater than zero. The 
means of the normalized skewness measured in elderly signals are inclined to be lesser than zero, that is negative values. In particular, in elderly signals, the mean of the normalized skewness is slightly broader than those of normal signals. As may be seen from Table 3, in statistics of the mean of the normalized skewness, the mean of normal signals (Female) is 0.131 and the means of elderly signals (Female) and (Male) are 0.002 and -0.197 , respectively. The range of normal signals (Female) is 0.53 and the range of elderly signals (Female) and (Male) are 0.93 and 0.64 , respectively. The $25^{\text {th }}, 50^{\text {th }}$, and $75^{\text {th }}$ percentiles are $-0.024,0.067$, and 0.290 , respectively, for the means of the normalized skewness measured in normal signal (Female). The $25^{\text {th }}, 50^{\text {th }}$, and $75^{\text {th }}$ percentiles are $0.121,-0.054$, and 0.226 , respectively, for the means of the normalized skewness measured in elderly signal (Female). Finally, the $25^{\text {th }}, 50^{\text {th }}$, and $75^{\text {th }}$ percentiles are -0.343 , -0.231 , and -0.095 , respectively, for the means of the normalized skewness estimated in elderly signal (Male). There is a clear difference between the distribution of the means of the normalized skewness for normal and elderly signals. In case of the mean of the normalized kurtosis, one estimated in normal signals is included in one estimated in elderly signals. It can be shown by the fact that the maximum, minimum, and range values of the mean of the normalized kurtosis estimated in normal signals are 5.46, 8.47, and 3.01, respectively, in shown as in Table 3. However, the mean of normal signals (Female) is 6.301 and the means of elderly signals (Female) and (Male) are 7.284 and 7.257 , respectively. It shows a clear difference between the normal and elderly signals. Similarly, it is obvious that the mean of the normalized skewness sufficiently analyze the normal and elderly signals and can be used for automatic classification between the normal and elderly signals.

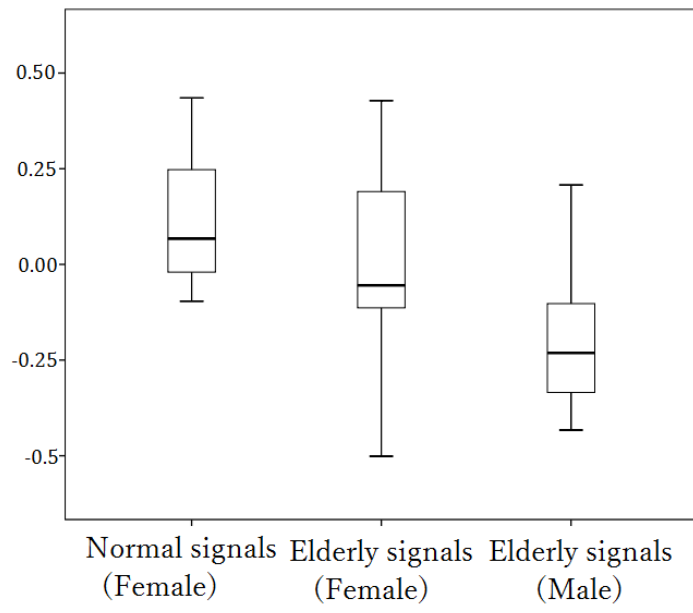

(a) The means of the normalized skewness

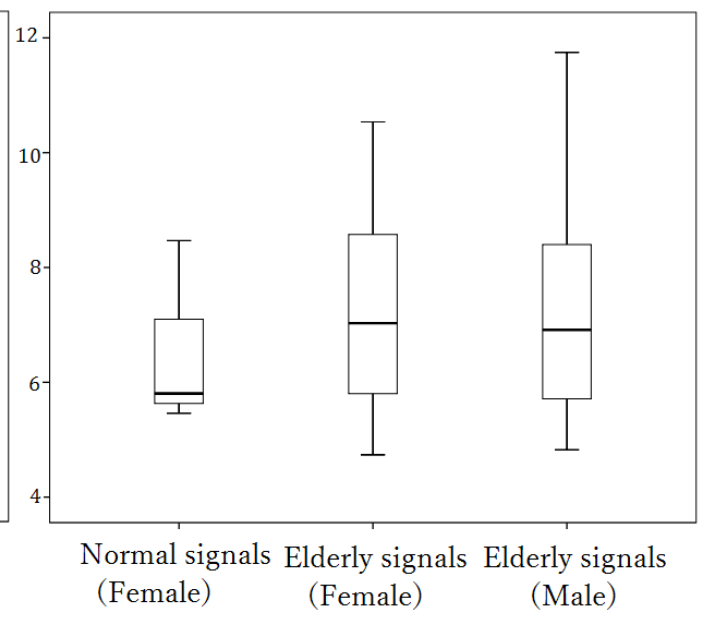

(b) The means of the normalized kurtosis

Figure 3. Distributions of the Means of the Normalized Skewness and Kurtosis

Table 3. Statistics of the Means of the Normalized Skewness and Kurtosis

\begin{tabular}{|c|c|c|c|}
\hline The means of normalized skewness & $\begin{array}{c}\text { Normal } \\
\text { signals } \\
\text { (Female) }\end{array}$ & $\begin{array}{c}\text { Elderly } \\
\text { signals } \\
\text { (Female) }\end{array}$ & $\begin{array}{c}\text { Elderly } \\
\text { signals } \\
\text { (Male) }\end{array}$ \\
\hline Mean & 0.131 & 0.002 & -0.197 \\
\hline Median & 0.067 & -0.054 & -0.231 \\
\hline Mode & -0.10 & -0.5 & -0.43 \\
\hline Range & 0.53 & 0.93 & 0.64 \\
\hline Minimum value & -0.10 & -0.5 & -0.43 \\
\hline
\end{tabular}




\begin{tabular}{|c|c|c|c|c|}
\hline \multicolumn{2}{|c|}{ Maximum value } & 0.44 & 0.43 & 0.21 \\
\hline \multirow{3}{*}{ Percentile } & 25 & -0.024 & -0.121 & -0.343 \\
\hline & 50 & 0.067 & -0.054 & -0.231 \\
\hline & 75 & 0.290 & 0.226 & -0.095 \\
\hline \multicolumn{2}{|c|}{ The means of normalized kurtosis } & $\begin{array}{c}\text { Normal } \\
\text { signals } \\
\text { (Female) }\end{array}$ & $\begin{array}{c}\text { Elderly } \\
\text { signals } \\
\text { (Female) }\end{array}$ & $\begin{array}{l}\text { Elderly } \\
\text { signals } \\
\text { (Male) } \\
\end{array}$ \\
\hline \multicolumn{2}{|c|}{ Mean } & 6.301 & 7.284 & 7.257 \\
\hline \multicolumn{2}{|c|}{ Median } & 5.804 & 7.030 & 6.913 \\
\hline \multicolumn{2}{|c|}{ Mode } & 5.46 & 4.74 & 4.82 \\
\hline \multicolumn{2}{|c|}{ Range } & 3.01 & 5.80 & 6.92 \\
\hline \multicolumn{2}{|c|}{ Minimum value } & 5.46 & 4.74 & 4.82 \\
\hline \multicolumn{2}{|c|}{ Maximum value } & 8.47 & 10.54 & 11.75 \\
\hline \multirow[t]{3}{*}{ Percentile } & 25 & 5.600 & 5.790 & 5.677 \\
\hline & 50 & 5.804 & 7.030 & 6.913 \\
\hline & 75 & 7.113 & 8.659 & 8.450 \\
\hline
\end{tabular}

Figure 4 shows the distributions of the RSK and their means in the form of box plot. There are similar dispersion among the RSK estimated from normal (Female), elderly (Female), and elderly (Male) signals. From statistics of the RSK in the Table 4, the mean of normal signals (Female) is 0.019 and the means of elderly signals (Female) and (Male) are 0.024 and 0.017 , respectively. The $25^{\text {th }}, 50^{\text {th }}$, and $75^{\text {th }}$ percentiles are $0.002,0.011$, and 0.030 , respectively, for the RSK measured in normal signal (Female). The $25^{\text {th }}, 50^{\text {th }}$, and $75^{\text {th }}$ percentiles are $0.003,0.013$, and 0.037 , respectively, for the RSK measured in elderly signal (Female). So, the RSK estimated in normal signals include the one estimated in elderly signals. There is little difference between the distributions of the RSK in normal and elderly signals. Also it is definitely shown in statistics of the means of RSK in Table 4.

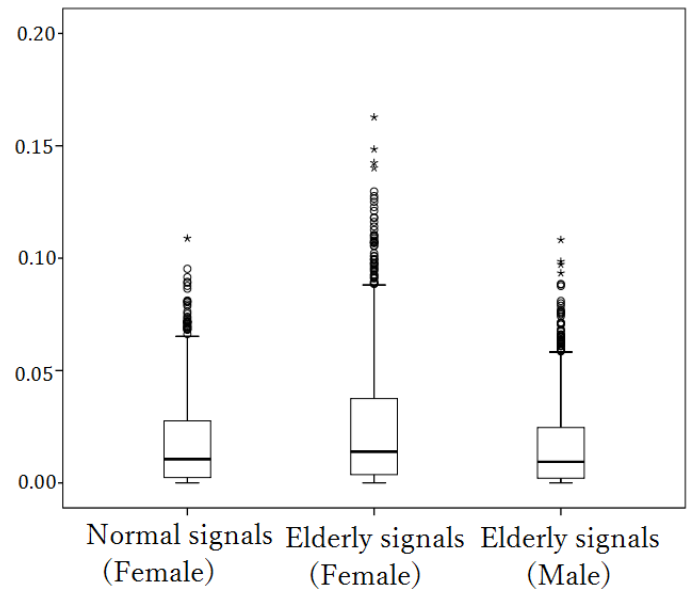

(a) The distribution of RSK

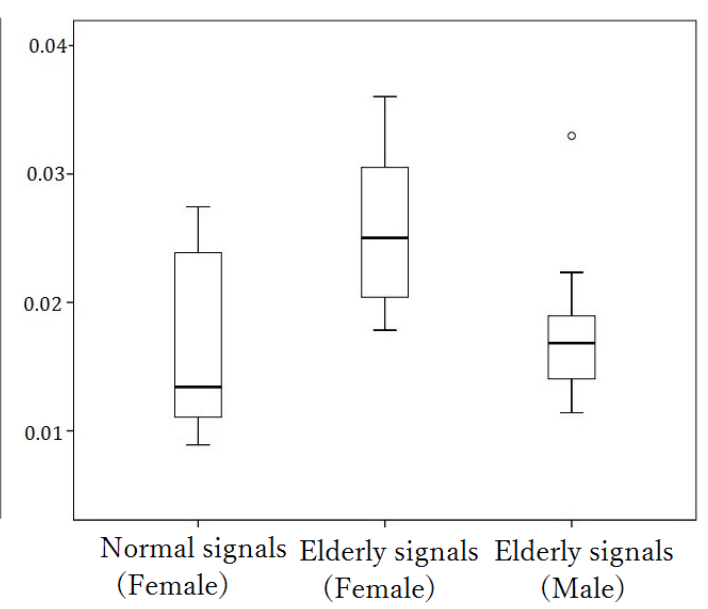

(b) The means of RSK

Figure 4. Distributions of the RSK and Their Means 
Table 4. Statistics of the RSK

\begin{tabular}{|c|c|c|c|c|}
\hline \multicolumn{2}{|c|}{ The RSK } & $\begin{array}{c}\text { Normal signals } \\
\text { (Female })\end{array}$ & $\begin{array}{l}\text { Elderly signals } \\
\text { (Female) }\end{array}$ & $\begin{array}{c}\text { Elderly signals } \\
\text { (Male) }\end{array}$ \\
\hline \multicolumn{2}{|c|}{ Mean } & 0.019 & 0.024 & 0.017 \\
\hline \multicolumn{2}{|c|}{ Median } & 0.011 & 0.013 & 0.009 \\
\hline \multicolumn{2}{|c|}{ Mode } & 0.00 & 0.00 & 0.00 \\
\hline \multicolumn{2}{|c|}{ Range } & 0.11 & 0.16 & 0.19 \\
\hline \multicolumn{2}{|c|}{ Minimum value } & 0.00 & 0.00 & 0.00 \\
\hline \multicolumn{2}{|c|}{ Maximum value } & 0.11 & 0.16 & 0.19 \\
\hline \multirow[t]{3}{*}{ Percentile } & 25 & 0.002 & 0.003 & 0.002 \\
\hline & 50 & 0.011 & 0.013 & 0.009 \\
\hline & 75 & 0.030 & 0.037 & 0.025 \\
\hline \multicolumn{2}{|c|}{ The means of RSK } & $\begin{array}{c}\text { Normal signals } \\
\text { (Female) }\end{array}$ & $\begin{array}{l}\text { Elderly signals } \\
\text { (Female) }\end{array}$ & $\begin{array}{l}\text { Elderly signals } \\
\text { (Male) }\end{array}$ \\
\hline \multicolumn{2}{|c|}{ Mean } & 0.017 & 0.025 & 0.018 \\
\hline \multicolumn{2}{|c|}{ Median } & 0.013 & 0.025 & 0.017 \\
\hline \multicolumn{2}{|c|}{ Mode } & 0.009 & 0.018 & 0.011 \\
\hline \multicolumn{2}{|c|}{ Range } & 0.019 & 0.018 & 0.021 \\
\hline \multicolumn{2}{|c|}{ Minimum value } & 0.009 & 0.018 & 0.011 \\
\hline \multicolumn{2}{|c|}{ Maximum value } & 0.027 & 0.036 & 0.033 \\
\hline \multirow[t]{3}{*}{ Percentile } & 25 & 0.011 & 0.020 & 0.014 \\
\hline & 50 & 0.013 & 0.025 & 0.017 \\
\hline & 75 & 0.024 & 0.030 & 0.020 \\
\hline
\end{tabular}

\subsection{Distributions of HOS-Parameters Estimated In Time Frequency}

Figure 5(a), (b), and (c) present clear distinctions between the bicoherence distributions of the different voice signals. In the bicoherence distribution of elderly signals, the greatest amplitude is observed, and the distribution tends towards higher values. Overall, the distributions become broader and more dispersed appearance with higher bicoherence values. In the bicoherence distribution of normal signals as shown in (a), the amplitudes tends to be lower values and a more assembled structure than those of the elderly signals.

Figure 6 shows the characteristics of the MB parameters estimated for normal and elderly signals. The MB values have significantly lower ones in case of normal signals, similarly to the behavior shown in Figure 5. These distributions also presents clear distinctions between normal and elderly signals. 


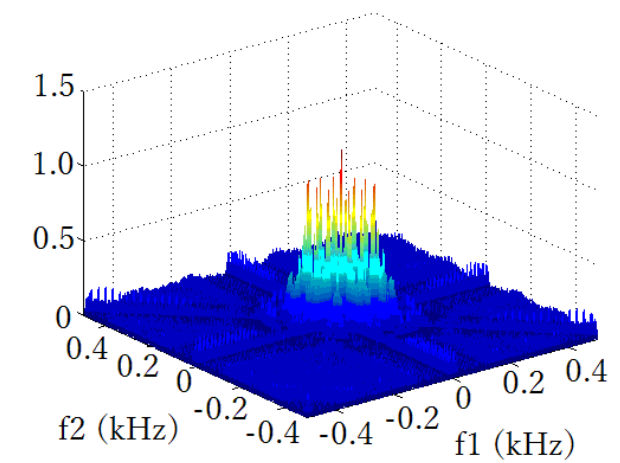

(a) Normal siganls (Female)

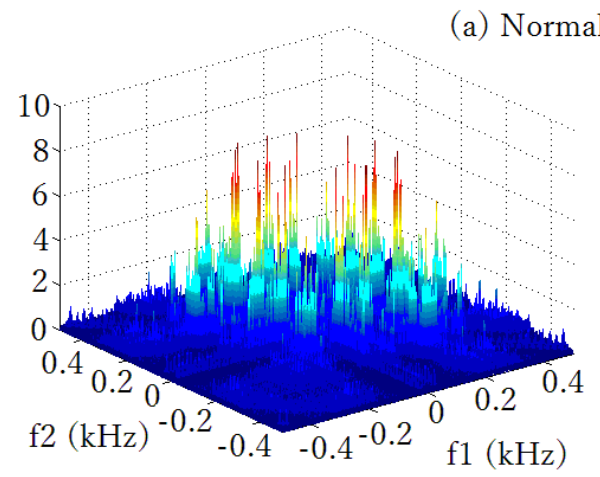

(b) Elderly signals (Female)

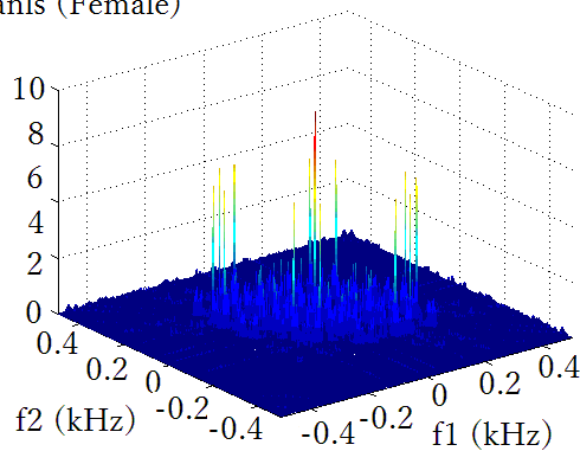

(c) Elderly signals (Male)

Figure 5. Bicoherence Distributions of Different Signals

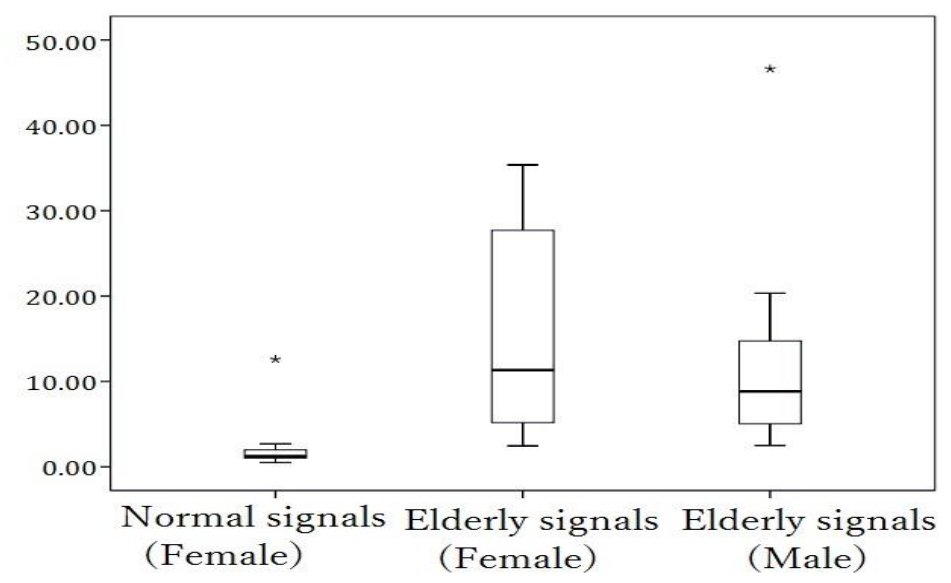

Figure 6. Distributions of MB parameter among Different Signals

\subsection{Statistical Analysis}

Table 5 shows the statistical analysis between normal and elderly voice signals. The purpose of a Mann-Whitney $U$ test is to determine if significant differences exist in the dependent variables such as the mean of normalized skewness, the mean of normalized kurtosis, RSK, and MB that are used for automatic classification between normal and elderly voice signals. In Table 5 , since the mean of normalized skewness and MB show p-values <.05, it is said that they are useful and meaningful for the classification of elderly voice signal. In particular, MB performs better statistically than do the other parameters. 
Table 6 shows the one way ANOVA analysis among normal, female elderly, and male elderly voice signals. Scheffe is used as post hoc test. The mean of normalized skewness, RSK, and MB are significant for the objective classification among three voice signals because they show p-values <.05. RSK specially performs better statistically than do the other parameters. The mean of normalized skewness is especially meaningful for the classification between normal and male elderly signals. The RSK and MB are particularly significant for the analysis between normal and female elderly signals.

Table 5. Statistical Analysis between Normal and Elderly Signals

\begin{tabular}{|c|c|}
\hline The mean of normalized skewness & $\mathrm{p}=0.006^{*}$ \\
\hline The mean of normalized kurtosis & $\mathrm{p}=0.104$ \\
\hline $\begin{array}{c}\text { The ratio of the normalized skewness and } \\
\text { kurtosis (RSK) }\end{array}$ & $\mathrm{p}=0.071$ \\
\hline Maximum Bicoherence (MB) & $\mathrm{p}=0.000^{*}$ \\
\hline
\end{tabular}

Table 6. Statistical Analysis among Normal, Female Elderly and Male Elderly Signals

\begin{tabular}{|c|c|c|c|}
\hline & $\begin{array}{c}\text { Normal } \\
\text { signals (Female) }\end{array}$ & $\begin{array}{l}\text { Elderly signals } \\
\text { (Female) }\end{array}$ & $\begin{array}{l}\text { Elderly signals } \\
\text { (Male) }\end{array}$ \\
\hline \multicolumn{4}{|c|}{ The mean of normalized skewness $\left(p=0.008^{*}\right)$} \\
\hline $\begin{array}{c}\text { Normal } \\
\text { signals (Female) }\end{array}$ & - & 0.421 & $0.008^{*}$ \\
\hline $\begin{array}{c}\text { Elderly } \\
\text { signals (Female) }\end{array}$ & - & - & 0.139 \\
\hline $\begin{array}{c}\text { Elderly } \\
\text { signals (Male) }\end{array}$ & - & - & - \\
\hline \multicolumn{4}{|c|}{ The mean of normalized kurtosis $(\mathrm{p}=0.334)$} \\
\hline $\begin{array}{c}\text { Normal } \\
\text { signals (Female) }\end{array}$ & - & 0.426 & 0.445 \\
\hline $\begin{array}{c}\text { Elderly } \\
\text { signals (Female) }\end{array}$ & - & - & 0.999 \\
\hline $\begin{array}{c}\text { Elderly } \\
\text { signals (Male) }\end{array}$ & - & - & - \\
\hline \multicolumn{4}{|c|}{ The ratio of the normalized skewness and kurtosis $(\mathrm{RSK})\left(\mathrm{p}=0.011^{*}\right)$} \\
\hline $\begin{array}{c}\text { Normal } \\
\text { signals (Female) }\end{array}$ & - & $0.018^{*}$ & 0.869 \\
\hline $\begin{array}{c}\text { Elderly } \\
\text { signals (Female) }\end{array}$ & - & - & 0.058 \\
\hline $\begin{array}{c}\text { Elderly } \\
\text { signals (Male) }\end{array}$ & - & - & - \\
\hline \multicolumn{4}{|c|}{ Maximum Bicoherence (MB) $(\mathrm{p}=0.02 *)$} \\
\hline $\begin{array}{c}\text { Normal } \\
\text { signals (Female) }\end{array}$ & - & $0.026^{*}$ & 0.107 \\
\hline $\begin{array}{c}\text { Elderly } \\
\text { signals (Female) }\end{array}$ & - & - & 0.790 \\
\hline $\begin{array}{c}\text { Elderly } \\
\text { signals (Male) }\end{array}$ & - & - & - \\
\hline
\end{tabular}




\section{Conclusions}

Speech interface is currently using an optimized method based on the average speech pattern of all aged people [2]-[3][11]-[12]. If it is a little larger deviation from an average value, it may have an effect on the performance degradation of voice analysis and recognition [14]-[15]. In case of most welfare smart devices, the elderly signals have been neglected due to interface which does not take into account the elderly [6]. Anatomy and physiological changes in the larynx due to aging may change the pitch of the voice and it can be distinguished from the normal signal as a part of speech disorder [2]-[5].

Many studies related to elderly signals have been based on the acoustic analysis of the voice samples including jitter and shimmer [3]-[4][6]-[8]. However, since these parameters are based on the fundamental frequency, a very reliable pitch extraction is necessary to measure voicing irregularities in elderly signals [12]. Recently, it is well known that higher-order statistics (HOS) which is not related to the pitch estimation is to be effective for the classification of signal type. Further, the combination of HOS analysis and the linear predictive coding (LPC) residual may help to effectively construct important information to distinguish between normal and elderly signals [13]-[17].

In this paper, the mean of normalized skewness, the mean of normalized kurtosis, the ratio of the normalized skewness and kurtosis (RSK), and maximum bicoherence (MB) are analyzed from LPC residual of normal and elderly signals. Distributions of the normalized skewness, the mean of the normalized skewness, the normalized kurtosis, the mean of the normalized kurtosis, the RSK, their means, bicoherence, and MB parameters are examined from normal, female elderly, and male elderly signals. The strong correlations between the HOS-based parameters and elderly signal are demonstrated.

The mean of normalized skewness and MB parameter are useful and meaningful in an analysis between normal and Elderly signals because they show p-values <.05. In particular, it is believed that MB is very significant for the classification between normal and elderly signals. One way ANOVA analysis is performed for various parameters estimated among normal, female elderly, and male elderly signals. The mean of normalized skewness, RSK, and MB are significant for the objective classification among three signals because they show p-values <.05. The mean of normalized skewness is especially meaningful for the classification between normal and male elderly signals. The RSK and MB are particularly significant for the analysis between normal and female elderly signals. Therefore, in most cases, MB is specially performs better statistically than do the other parameters.

These results will contribute to enhance the speech recognition performance of the existing smart medical systems for the elderly. This is also expected to help provide an easy access means for the elderly and the disabled who can be excluded from the rapid socialization.

\section{Acknowledgments}

This research was supported by the Basic Science Research Program through the National Research Foundation of Korea (NRF) funded by the Ministry of Science, ICT \& Future Planning (No. 2014-00540001).

\section{References}

[1] J. Lee, "KHIDI Brief”, Korea Health Industry Development Institute, vol.140, (2014), pp. 1-2.

[2] I. C. Kim and D. Y. Kim, "A Study on the Features for Building Korean Digit Recognition System Based on Multilayer Perceptron", Journal of the Korea Industrial Information Systems Research, vol. 6, no. 4, (2001), pp. 389-391.

[3] Y. k. Song, "Prevalence of Voice Disorders and Characteristics of Korean Voice Handicap Index in the Elderly", Journal of the Korean society of speech science, vol. 4, no. 3, (2012), pp. 151-159. 
[4] S. J. Lee and S. I. Kwon, "Elderly Speech Analysis for Improving Elderly Speech Recognition", Journal of Korean Institute of Information Scientists and Engineers, vol. 32, no. 11, (2014), pp. 16-20.

[5] J. C. Kahane, "Anatomic and physiologic changes in the aging peripheral speech mechanism", Edited D. S. Beasley and G. A. Davis, Grune \& Stratton, New York, (1981), pp. 21-45.

[6] S.Y. Lee, Editor, "The overall speaking rate and articulation rate of normal elderly people", Graduate program in speech and language pathology, Master these, Yonsei University, (2011).

[7] R. T. Sataloff, D. C. Rogen, M. Hawkshaw and J. R. Spiegel, "The three ages of voice. The aging adult voice", Journal of Voice, vol. 11, no. 2, (1997), pp. 156-160.

[8] S. Lee and S. Kim, "Elderly speech analysis for improving elderly speech recognition", Communications of the KOREA Information Science Society, vol. 32, no. 11, (2014), pp. 15-20.

[9] S. H. Kim and D. H. Ko, "Fundamental frequencies in Korean elderly speakers", Speech science, vol. 15, no. 3, (2008), pp. 95-102.

[10] J. Y. Lee and S. H. Choi, "Perturbation analysis using a moving window for disordered voices", International Journal of Engineering, Science, and Innovative Technology, vol. 3, no. 1, (2012), pp. 1-10.

[11] J. Y. Lee, S. H. Choi, J. J. Jiang, M. S. Hahn and H. S, Choi, "Perturbation and Perceptual Analysis of Pathological Sustained Vowels According to Signal Typing”, Malsori and Speech Science, vol. 2, no. 2, (2010), pp. 109-115.

[12] J. Y. Lee, "Fundamental Frequency Characteristics using Moving Window Method for Korean Elderly Voices", International Journal of Engineering and Technology., vol. 8, no. 3, (2016), pp. 1589-1599.

[13] J. B. Alonso, J. de Leon, I. Alonso and M. A. Ferrer, "Automatic Detection of Pathologies in the Voice by HOS Based Parameters", EURASIP Journal on Applied Signal Processing, vol. 4, (2001), pp. 275284.

[14] J. Y. Lee, S. Jeong and M. S. Hahn, "Pathological Voice Detection Using Efficient Combination of Heterogeneous Features", IEICE Transactions on Information and Systems, vol. E91-D, no. 2, (2008), pp. 367-370.

[15] J. Y. Lee, S. Jeong, H. S. Choi and M. S. Hahn, "Objective pathological voice quality assessment based on HOS features", IEICE Transactions on Information and Systems., vol. E91-D, no. 12, (2008), pp. 2888-2891.

[16] J. Y. Lee, "A two-stage approach using Gaussian mixture models and higher-order statistics for a classification of normal and pathological voices", Advances in Signal Processing on Euraship, vol. 252, (2012), http://asp.eurasipjournals.com/content/2012/1/252.

[17] J. Y. Lee and M. S. Hahn, "Automatic Assessment of Pathological Voice Quality Using Higher-Order Statistics in the LPC Residual Domain", EURASIP Journal on Advances in Signal Processing, (2009), Article ID 748207.

[18] J. Y. Lee, S. B. Jeong, M. S. Hahn, A. Sprecher and J. J. Jiang, “An efficient approach using HOS-based parameters in the LPC residual domain to classify breathy and rough voices", Biomedical Signal Processing and Control., vol. 6, no. 2, (2011), pp. 186-196.

[19] E. Nemer, R. Goubran and S. Goubran, "Robust voice activity detection using higher-order statistics in the LPC residual domain", IEEE Transaction Speech and Audio Processing., vol. 9, no. 3, (2001), pp. 217-231.

[20] M. de Oliveira Rosa, J. C. Pereira and M. Grellet, "Adaptive estimation of residual signal for voice pathology diagnosis", IEEE Transaction on Biomedical Engineering, vol. 47, no. 1, (2000), pp. 96-104.

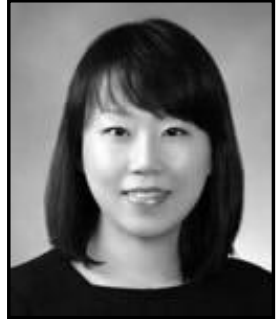

Ji Yeoun Lee, she obtained B.S. in division of Electronics and Computer Engineering from Hanyang University in the year of 2001, M.S. and Ph.D in Electrical Engineering from Korea Advanced Institute of Science and Technology (KAIST) in the year of 2003 and 2008. Dr. JiYeoun Lee is now an assistant professor at the faculty of Biomedical Engineering, Jungwon University. Her research interest fields are Biomedical Signal Processing, Noise Reduction, Sound Localization, and Audio Signal Processing. 
International Journal of Control and Automation

Vol. 10, No. 2 (2017) 